Appl. Set-Valued Anal. Optim. 3 (2021), No. 2, pp. 203-214

Available online at http://asvao.biemdas.com

https://doi.org/10.23952/asvao.3.2021.2.05

\title{
ON A VISCOSITY ITERATIVE ALGORITHM FOR VARIATIONAL INCLUSION PROBLEMS AND THE FIXED POINT PROBLEM OF COUNTABLY MANY NONEXPANSIVE MAPPINGS
}

\author{
LU-CHUAN CENG \\ Department of Mathematics, Shanghai Normal University, Shanghai 200234, China
}

\begin{abstract}
In this paper, we propose a viscosity iterative algorithm for finding a common solution of a common fixed point problem of a countable family of nonexpansive mappings and two variational inclusion problems. We investigate the convergence of the proposed algorithm and a strong convergence theorem is established in the setting of Banach spaces.

Keywords. Accretive operator; Nonexpansive mapping; Variational inclusion; Viscosity iterative algorithm.
\end{abstract}

\section{INTRODUCTION-PRELIMINARIES}

Let $E$ be a real Banach space with the dual $E^{*}$, and let $\emptyset \neq C \subset E$ be a closed convex set. Let $T$ be a mapping on $C$. We use the symbol $\operatorname{Fix}(T)$ to denote the set of fixed point of $T$. Recall that $T$ is said to be contractive if there exists a constant $\rho \in(0,1)$ such that $\|T x-T y\| \leq$ $\rho\|x-y\|, \forall x, y \in C$. $T$ is said to be nonexpansive if $\|T x-T y\| \leq\|x-y\|, \forall x, y \in C$. The class of nonexpansive mappings has many real applications in various optimization problems; see, e.g., $[1,2,3,4,5,6]$ and the references therein.

Recall that the generalized duality mapping, $J_{q}(q>1)$, from $E$ into $2^{E^{*}}$ is defined by

$$
J_{q}(x):=\left\{x^{*} \in E^{*}:\left\langle x^{*}, x\right\rangle=\|x\|^{q},\left\|x^{*}\right\|=\|x\|^{q-1}\right\}, \quad \forall x \in E,
$$

where $\langle\cdot, \cdot\rangle$ denotes the duality pairing between $E$ and $E^{*}$. In particular, $J_{2}$ is called the normalized duality mapping and it is usually denoted by $J$. One knows that $J_{q}(x)=\|x\|^{q-2} J(x)$ for all $x \neq 0$.

The modulus of convexity of $E$ is the function $\delta_{E}:(0,2] \rightarrow[0,1]$ defined by

$$
\delta_{E}(\varepsilon)=\inf \left\{1-\frac{\|x+y\|}{2}: x, y \in E,\|x\|=\|y\|=1,\|x-y\| \geq \varepsilon\right\} .
$$

The modulus of smoothness of $E$ is the function $\rho_{E}: R_{+}:=[0, \infty) \rightarrow R_{+}$defined by

$$
\rho_{E}(\tau)=\sup \left\{\frac{\|x+\tau y\|+\|x-\tau y\|}{2}-1: x, y \in E,\|x\|=\|y\|=1\right\} .
$$

A Banach space $E$ is said to be uniformly convex if $\delta_{E}(\varepsilon)>0, \forall \varepsilon \in(0,2]$. It is said to be uniformly smooth if $\lim _{\tau \rightarrow 0^{+}} \frac{\rho_{E}(\tau)}{\tau}=0$. Also, it is said to be $q$-uniformly smooth with $q>1$

E-mail address: zenglc@ @shu.edu.cn.

Received January 5, 2021; Accepted March 28, 2021.

(C)2021 Applied Set-Valued Analysis and Optimization 
if $\exists c>0$ such that $\rho_{E}(t) \leq c t^{q}, \forall t>0$. If $E$ is $q$-uniformly smooth, then $q \leq 2$. It is known that sequence space $\ell_{p}$ and Lebesgue space $L_{p}$ are $\min \{p, 2\}$-uniformly smooth for every $p>1$ $[7,8,9]$.

Let $B: C \rightarrow 2^{E}$ be a set-valued operator with $B x \neq \emptyset, \forall x \in C$. Let $q>1$. An operator $B$ is said to be accretive if, for each $x, y \in C, \exists j_{q}(x-y) \in J_{q}(x-y)$ such that $\left\langle u-v, j_{q}(x-y)\right\rangle \geq 0$, $\forall u \in B x, v \in B y$. Further, $B$ is said to be $m$-accretive if $(I+\lambda B) C=E$ for all $\lambda>0$. For an $m$-accretive operator $B$, we can define the mapping $J_{\lambda}^{B}:(I+\lambda B) C \rightarrow C$ by $J_{\lambda}^{B}=(I+\lambda B)^{-1}$ for each $\lambda>0$. Such $J_{\lambda}^{B}$ is called the resolvent of $B$ for $\lambda>0$. In the sequel, we will use the notation $T_{\lambda}:=J_{\lambda}^{B}(I-\lambda A)=(I+\lambda B)^{-1}(I-\lambda A), \forall \lambda>0$. From Barbu [10], one has the following resolvent identity: $J_{\lambda}^{B} x=J_{\mu}^{B}\left(\frac{\mu}{\lambda} x+\left(1-\frac{\mu}{\lambda}\right) J_{\lambda}^{B} x\right), \forall \lambda, \mu>0, x \in E$. An accretive operator $B$ is said to be $\alpha$-inverse-strongly accretive of order $q$ if, for each $x, y \in C$, there exists $j_{q}(x-y) \in J_{q}(x-y)$ such that $\left\langle u-v, j_{q}(x-y)\right\rangle \geq \alpha\|u-v\|^{q}, \forall u \in B x, v \in B y$ for some $\alpha>0$. If $E=H$ a Hilbert space, then $B$ is called $\alpha$-inverse-strongly monotone.

In 2017, Chang, Wen and Yao [11] suggested a generalized viscosity implicit rule for finding a point in $(A+B)^{-1} 0$, where $A: E \rightarrow E$ is an $\alpha$-inverse-strongly accretive mapping of order $q$ and $B: E \rightarrow 2^{E}$ is an $m$-accretive operator:

$$
x_{j+1}=\alpha_{j} f\left(x_{j}\right)+\left(1-\alpha_{j}\right) J_{\lambda}^{B}(I-\lambda A)\left(t_{j} x_{j}+\left(1-t_{j}\right) x_{j+1}\right), \quad \forall j \geq 1,
$$

where $f$ is a contractive mapping, $J_{\lambda}^{B}=(I+\lambda B)^{-1}, \lambda \in(0, \infty)$ and the sequences $\left\{t_{j}\right\},\left\{\alpha_{j}\right\} \subset$ $(0,1)$ are such that (i) $\lim _{j \rightarrow \infty} \alpha_{j}=0, \sum_{j=1}^{\infty} \alpha_{j}=\infty$; (ii) $\sum_{j=1}^{\infty}\left|\alpha_{j+1}-\alpha_{j}\right|<\infty$; (iii) $0<\varepsilon \leq$ $t_{j} \leq t_{j+1}<1$; (iv) $0<\lambda \leq\left(\frac{\alpha q}{\kappa_{q}}\right)^{\frac{1}{q-1}}$. They proved the strong convergence of $\left\{x_{j}\right\}$ to a point of $(A+B)^{-1} 0$, which solves some generalized variational inequality.

Recently, Sunthrayuth and Cholamjiak [12] proposed the following modified viscosity-type extragradient method for a fixed point problem of a nonexpansive mapping and solution of the inclusion problem of an $\alpha$-inverse-strongly accretive mapping $A$ and an $m$-accretive operator $B$

$$
\left\{\begin{array}{l}
y_{j}=J_{\lambda_{j}}^{B}\left(x_{j}-\lambda_{j} A x_{j}\right), \\
z_{j}=J_{\lambda_{j}}^{B}\left(x_{j}-\lambda_{j} A y_{j}+r_{j}\left(y_{j}-x_{j}\right)\right), \\
x_{j+1}=\alpha_{j} f\left(x_{j}\right)+\beta_{j} x_{j}+\gamma_{j} S z_{j}, \quad \forall j \geq 0,
\end{array}\right.
$$

where $S$ is a nonexpansive mapping on $C, J_{\lambda_{j}}^{B}=\left(I+\lambda_{j} B\right)^{-1},\left\{r_{j}\right\},\left\{\alpha_{j}\right\},\left\{\beta_{j}\right\},\left\{\gamma_{j}\right\} \subset(0,1)$ and $\left\{\lambda_{j}\right\} \subset(0, \infty)$ are such that: (i) $\alpha_{j}+\beta_{j}+\gamma_{j}=1$; (ii) $\lim _{j \rightarrow \infty} \alpha_{j}=0, \sum_{j=1}^{\infty} \alpha_{j}=\infty$; (iii) $\left\{\beta_{j}\right\} \subset[a, b] \subset(0,1)$; and (iv) $0<\lambda \leq \lambda_{j}<\lambda_{j} / r_{j} \leq \mu<\left(\alpha q / \kappa_{q}\right)^{1 /(q-1)}, 0<r \leq r_{j}<1$. They proved the strong convergence of $\left\{x_{j}\right\}$ to a point of $\operatorname{Fix}(S) \cap(A+B)^{-1} 0$, which solves some generalized variational inequality.

In this paper, we consider the variational inclusion with two accretive operators and a common fixed point problem of a countable family of nonexpansive mappings. A viscosity method is introduced and the strong convergence of the suggested method is obtained in a uniformly convex and $q$-uniformly smooth Banach space with $q \in(1,2]$. Our results improve and extend the corresponding results in $[11,12,13,14,15,16,17]$ to a certain extent.

For obtain our main convergence theorem, we need the following lemmas.

Lemma 1.1. [13] Let $E$ be a q-uniformly smooth Banach space with $q \in(1,2]$. Let $A: C \rightarrow E$ be an $\alpha$-inverse-strongly accretive mapping of order $q$. Then, for any given $\lambda \geq 0$,

$$
\|(I-\lambda A) x-(I-\lambda A) y\|^{q} \leq\|x-y\|^{q}-\lambda\left(\alpha q-\kappa_{q} \lambda^{q-1}\right)\|A x-A y\|^{q}, \quad \forall x, y \in C,
$$


where $\kappa_{q}>0$ is the $q$-uniform smoothness coefficient of $E$. In particular, if $0 \leq \lambda \leq\left(\frac{\alpha q}{\kappa_{q}}\right)^{\frac{1}{q-1}}$, then $I-\lambda A$ is nonexpansive.

Lemma 1.2. [9] Let $q \in(1,2]$ a fixed real number and let $E$ be q-uniformly smooth. Then $\| x+$ $y\left\|^{q} \leq\right\| x\left\|^{q}+q\left\langle y, J_{q}(x)\right\rangle+\kappa_{q}\right\| y \|^{q}, \forall x, y \in E$, where $\kappa_{q}$ is the q-uniform smoothness coefficient of $E$.

Lemma 1.3. $[9,16]$ Let $q>1$ and $r>0$ be two fixed real numbers and let $E$ be uniformly convex. Let $B_{r}:=\{x \in E:\|x\| \leq r\}$. Then there exist strictly increasing, continuous and convex functions $g, h: R_{+} \rightarrow R_{+}$with $g(0)=0$ and $h(0)=0$ such that

(a) $\|\mu x+(1-\mu) y\|^{q} \leq \mu\|x\|^{q}+(1-\mu)\|y\|^{q}-\mu(1-\mu) g(\|x-y\|)$ for all $x, y \in B_{r}$ and $\mu \in[0,1]$

(b) $\|\lambda x+\mu y+v z\|^{q} \leq \lambda\|x\|^{q}+\mu\|y\|^{q}+v\|z\|^{q}-\lambda \mu g(\|x-y\|)$ for all $x, y, z \in B_{r}$ and $\lambda, \mu, v \in$ $[0,1]$ with $\lambda+\mu+v=1$.

(c) $h(\|x-y\|) \leq\|x\|^{q}-q\left\langle x, j_{q}(y)\right\rangle+(q-1)\|y\|^{q}$ for all $x, y \in B_{r}$ and $j_{q}(y) \in J_{q}(y)$.

Lemma 1.4. [18] Let $\left\{S_{n}\right\}_{n=0}^{\infty}$ be a sequence of self-mappings on $C$ such that $\sum_{n=1}^{\infty} \sup _{x \in C} \| S_{n} x-$ $S_{n-1} x \|<\infty$. Then for each $y \in C,\left\{S_{n} y\right\}$ converges strongly to some point of $C$. Moreover, let $S$ be a self-mapping on $C$ defined by $S y=\lim _{n \rightarrow \infty} S_{n} y, \forall y \in C$. Then $\lim _{n \rightarrow \infty} \sup _{x \in C}\left\|S_{n} x-S x\right\|=$ 0 .

Lemma 1.5. $[13,19]$ The following statements hold:

(i) $\operatorname{Fix}\left(T_{\lambda}\right)=(A+B)^{-1} 0, \forall \lambda>0$;

(ii) $\left\|y-T_{\lambda} y\right\| \leq 2\left\|y-T_{r} y\right\|$ for $0<\lambda \leq r$ and $y \in C$.

Lemma 1.6. [20] Let $E$ be strictly convex, and let $\left\{S_{n}\right\}_{n=0}^{\infty}$ be a sequence of nonexpansive mappings on $C$. Suppose that $\bigcap_{n=0}^{\infty} \operatorname{Fix}\left(S_{n}\right)$ is nonempty. Let $\left\{\lambda_{n}\right\}$ be a sequence of positive numbers with $\sum_{n=0}^{\infty} \lambda_{n}=1$. Then a mapping $S$ on $C$ defined by $S x=\sum_{n=0}^{\infty} \lambda_{n} S_{n} x, \forall x \in C$ is defined well, nonexpansive and $\operatorname{Fix}(S)=\bigcap_{n=0}^{\infty} \operatorname{Fix}\left(S_{n}\right)$ holds.

Lemma 1.7. [21] Let $E$ be uniformly smooth, $T: C \rightarrow C$ a nonexpansive mapping with $\operatorname{Fix}(T) \neq$ $\emptyset$ and $f: C \rightarrow C$ a fixed contraction. For each $t \in(0,1)$, let $z_{t} \in C$ be the unique fixed point of the contraction $C \ni z \mapsto t f(z)+(1-t) T z$ on $C$, i.e., $z_{t}=t f\left(z_{t}\right)+(1-t) T z_{t}$. Then $\left\{z_{t}\right\}$ converges strongly to a fixed point $x^{*} \in \operatorname{Fix}(T)$, which solves $\left\langle(I-f) x^{*}, J\left(x^{*}-x\right)\right\rangle \leq 0, \forall x \in \operatorname{Fix}(T)$.

Lemma 1.8. [22] Let $\left\{a_{n}\right\}$ be a sequence in $[0, \infty)$ such that $a_{n+1} \leq\left(1-s_{n}\right) a_{n}+s_{n} v_{n}, \forall n \geq 0$, where $\left\{s_{n}\right\}$ and $\left\{v_{n}\right\}$ satisfy the conditions: (i) $\left\{s_{n}\right\} \subset[0,1], \sum_{n=0}^{\infty} s_{n}=\infty$; (ii) $\limsup _{n \rightarrow \infty} v_{n} \leq 0$ or $\sum_{n=0}^{\infty}\left|s_{n} v_{n}\right|<\infty$. Then $\lim _{n \rightarrow \infty} a_{n}=0$.

Lemma 1.9. [23] Let $\left\{\Gamma_{n}\right\}$ be a sequence of real numbers that does not decrease at infinity in the sense that there exists a subsequence $\left\{\Gamma_{n_{i}}\right\}$ of $\left\{\Gamma_{n}\right\}$ which satisfies $\Gamma_{n_{i}}<\Gamma_{n_{i}+1}$ for each integer $i \geq 1$. Define the sequence $\{\tau(n)\}_{n \geq n_{0}}$ of integers as follows:

$$
\tau(n)=\max \left\{k \leq n: \Gamma_{k}<\Gamma_{k+1}\right\},
$$

where integer $n_{0} \geq 1$ such that $\left\{k \leq n_{0}: \Gamma_{k}<\Gamma_{k+1}\right\} \neq \emptyset$. Then, the following hold:

(i) $\tau\left(n_{0}\right) \leq \tau\left(n_{0}+1\right) \leq \cdots$ and $\tau(n) \rightarrow \infty$;

(ii) $\Gamma_{\tau(n)} \leq \Gamma_{\tau(n)+1}$ and $\Gamma_{n} \leq \Gamma_{\tau(n)+1} \forall n \geq n_{0}$. 


\section{MAIN RESUlts}

From now on, we always suppose that $C$ is a nonempty closed convex subset of a uniformly convex and $q$-uniformly smooth Banach space $E$ with $q \in(1,2]$. Let $B, M: C \rightarrow 2^{E}$ be $m$ accretive operators, and let $A, F: C \rightarrow E$ be $\alpha$-inverse-strongly accretive mapping of order $q$ and $\beta$-inverse-strongly accretive mapping of order $q$, respectively. Let $f: C \rightarrow C$ be a $\rho$-contraction with constant $\rho \in\left[0, \frac{1}{q}\right)$, and let $\left\{S_{n}\right\}_{n=0}^{\infty}$ be a countable family of nonexpansive self-mappings on $C$. Assume that $\Omega:=\bigcap_{n=0}^{\infty} \operatorname{Fix}\left(S_{n}\right) \cap(A+B)^{-1} 0 \cap(F+M)^{-1} 0 \neq \emptyset$.

Algorithm 2.1. Initial Step. Give $\zeta \in(0,1)$ and $x_{0} \in C$.

Iteration Steps. Given the current iterate $x_{n}$, compute $x_{n+1}$ as follows:

Step 1. Calculate $v_{n}=J_{\lambda_{n}}^{B}\left(x_{n}-\lambda_{n} A x_{n}\right)$;

Step 2. Calculate $u_{n}=J_{\lambda_{n}}^{B}\left(x_{n}-\lambda_{n} A v_{n}+r_{n}\left(v_{n}-x_{n}\right)\right)$;

Step 3. Calculate $x_{n+1}=(1-\zeta) S_{n} u_{n}+\zeta J_{\mu_{n}}^{M}\left(\alpha_{n} f\left(u_{n}\right)+\left(1-\alpha_{n}\right) u_{n}-\mu_{n} F u_{n}\right)$, where $\left\{r_{n}\right\}$, $\left\{\alpha_{n}\right\} \subset(0,1)$ and $\left\{\lambda_{n}\right\},\left\{\mu_{n}\right\} \subset(0, \infty)$.

Set $n:=n+1$ and go to Step 1 .

Lemma 2.1. If $\left\{x_{n}\right\}$ is the sequence generated by Algorithm 2.1, then it is bounded.

Proof. Fix $p \in \Omega$. Then

$$
\begin{aligned}
p=S_{n} p & =J_{\lambda_{n}}^{B}\left(p-\lambda_{n} A p\right)=J_{\lambda_{n}}^{B}\left(\left(1-r_{n}\right) p+r_{n}\left(p-\frac{\lambda_{n}}{r_{n}} A p\right)\right) \\
& =J_{\mu_{n}}^{M}\left(p-\mu_{n} F p\right)=J_{\mu_{n}}^{M}\left(\alpha_{n} p+\left(1-\alpha_{n}\right)\left(p-\frac{\mu_{n}}{1-\alpha_{n}} F p\right)\right) .
\end{aligned}
$$

Using Lemmas 1.1, we have

$$
\begin{aligned}
\left\|v_{n}-p\right\|^{q} & \leq\left\|\left(I-\lambda_{n} A\right) x_{n}-\left(I-\lambda_{n} A\right) p\right\|^{q} \\
& \leq\left\|x_{n}-p\right\|^{q}-\lambda_{n}\left(\alpha q-\kappa_{q} \lambda_{n}^{q-1}\right)\left\|A x_{n}-A p\right\|^{q}
\end{aligned}
$$

which hence leads to $\left\|v_{n}-p\right\| \leq\left\|x_{n}-p\right\|$. By the convexity of $\|\cdot\|^{q}$, we deduce that

$$
\begin{aligned}
& \left\|u_{n}-p\right\|^{q} \\
& \leq\left\|\left(\left(1-r_{n}\right) x_{n}+r_{n}\left(v_{n}-\frac{\lambda_{n}}{r_{n}} A v_{n}\right)\right)-\left(\left(1-r_{n}\right) p+r_{n}\left(p-\frac{\lambda_{n}}{r_{n}} A p\right)\right)\right\|^{q} \\
& \leq\left(1-r_{n}\right)\left\|x_{n}-p\right\|^{q}+r_{n}\left\|\left(I-\frac{\lambda_{n}}{r_{n}} A\right) v_{n}-\left(I-\frac{\lambda_{n}}{r_{n}} A\right) p\right\|^{q} \\
& \leq\left(1-r_{n}\right)\left\|x_{n}-p\right\|^{q}+r_{n}\left[\left\|v_{n}-p\right\|^{q}-\frac{\lambda_{n}}{r_{n}}\left(\alpha q-\frac{\kappa_{q} \lambda_{n}^{q-1}}{r_{n}^{q-1}}\right)\left\|A v_{n}-A p\right\|^{q}\right] \\
& \leq\left(1-r_{n}\right)\left\|x_{n}-p\right\|^{q}+r_{n}\left[\left\|x_{n}-p\right\|^{q}-\lambda_{n}\left(\alpha q-\kappa_{q} \lambda_{n}^{q-1}\right)\left\|A x_{n}-A p\right\|^{q}\right. \\
& \left.-\frac{\lambda_{n}}{r_{n}}\left(\alpha q-\frac{\kappa_{q} \lambda_{n}^{q-1}}{r_{n}^{q-1}}\right)\left\|A v_{n}-A p\right\|^{q}\right] \\
& =\left\|x_{n}-p\right\|^{q}-r_{n} \lambda_{n}\left(\alpha q-\kappa_{q} \lambda_{n}^{q-1}\right)\left\|A x_{n}-A p\right\|^{q}-\lambda_{n}\left(\alpha q-\frac{\kappa_{q} \lambda_{n}^{q-1}}{r_{n}^{q-1}}\right)\left\|A v_{n}-A p\right\|^{q} .
\end{aligned}
$$

This ensures that $\left\|u_{n}-p\right\| \leq\left\|x_{n}-p\right\|$. We now put

$$
y_{n}:=J_{\mu_{n}}^{M}\left(\alpha_{n} f\left(u_{n}\right)+\left(1-\alpha_{n}\right) u_{n}-\mu_{n} F u_{n}\right)
$$


for all $n \geq 0$. Since $J_{\mu_{n}}^{M}$ and $I-\frac{\mu_{n}}{1-\alpha_{n}} F$ are nonexpansive for all $n \geq 0$, we obtain that

$$
\begin{aligned}
\left\|y_{n}-p\right\| & \leq\left\|\left(\alpha_{n} f\left(u_{n}\right)+\left(1-\alpha_{n}\right)\left(u_{n}-\frac{\mu_{n}}{1-\alpha_{n}} F u_{n}\right)\right)-\left(\alpha_{n} p+\left(1-\alpha_{n}\right)\left(p-\frac{\mu_{n}}{1-\alpha_{n}} F p\right)\right)\right\| \\
& \leq\left(1-\alpha_{n}\right)\left\|u_{n}-p\right\|+\alpha_{n}\left\|f\left(u_{n}\right)-f(p)\right\|+\alpha_{n}\|f(p)-p\| \\
& \leq\left(1-\alpha_{n}(1-\rho)\right)\left\|u_{n}-p\right\|+\alpha_{n}\|f(p)-p\| \\
& \leq\left(1-\alpha_{n}(1-\rho)\right)\left\|x_{n}-p\right\|+\alpha_{n}\|f(p)-p\| \\
& \leq \max \left\{\left\|x_{n}-p\right\|, \frac{\|f(p)-p\|}{1-\rho}\right\} .
\end{aligned}
$$

Hence, from the nonexpansivity of $S_{n}$, we get

$$
\begin{aligned}
\left\|x_{n+1}-p\right\| & \leq(1-\zeta)\left\|S_{n} u_{n}-p\right\|+\zeta\left\|y_{n}-p\right\| \\
& \leq(1-\zeta)\left\|x_{n}-p\right\|+\zeta \max \left\{\left\|x_{n}-p\right\|, \frac{\|f(p)-p\|}{1-\rho}\right\} \\
& \leq \max \left\{\left\|x_{n}-p\right\|, \frac{\|f(p)-p\|}{1-\rho}\right\} .
\end{aligned}
$$

By induction, we have

$$
\left\|x_{n}-p\right\| \leq \max \left\{\left\|x_{0}-p\right\|, \frac{\|f(p)-p\|}{1-\rho}\right\}, \quad \forall n \geq 0
$$

Consequently, $\left\{x_{n}\right\}$ is bounded, and so are $\left\{u_{n}\right\}\left\{v_{n}\right\},\left\{y_{n}\right\},\left\{S_{n} u_{n}\right\},\left\{A x_{n}\right\},\left\{A v_{n}\right\}$ and $\left\{F u_{n}\right\}$. This completes the proof.

Theorem 2.1. Let $\left\{x_{n}\right\}$ be the sequence generalized by Algorithm 2.1. Suppose that the following conditions hold:

(C1) $\lim _{n \rightarrow \infty} \alpha_{n}=0$ and $\sum_{n=0}^{\infty} \alpha_{n}=\infty$;

(C2) $0<r \leq r_{n}<1$ and $0<\lambda \leq \lambda_{n}<\frac{\lambda_{n}}{r_{n}} \leq \bar{\lambda}<\left(\frac{\alpha q}{\kappa_{q}}\right)^{\frac{1}{q-1}}$;

(C3) $0<a \leq \frac{\mu_{n}}{1-\alpha_{n}} \leq b<\left(\frac{\beta q}{\kappa_{q}}\right)^{\frac{1}{q-1}}$.

Assume that $\sum_{n=0}^{\infty} \sup _{x \in D}\left\|S_{n+1} x-S_{n} x\right\|<\infty$ for any bounded subset $D$ of $C$. Let $S: C \rightarrow C$ be a mapping defined by $S x=\lim _{n \rightarrow \infty} S_{n} x \forall x \in C$, and suppose that $\operatorname{Fix}(S)=\bigcap_{n=0}^{\infty} \operatorname{Fix}\left(S_{n}\right)$. Then $x_{n} \rightarrow x^{*} \in \Omega$.

Proof. Fix $x^{*} \in \Omega$. Using Lemma 1.1 and Lemma 1.2, we get

$$
\begin{aligned}
& \left\|y_{n}-x^{*}\right\|^{q} \\
& \leq\left\|\left(1-\alpha_{n}\right)\left(\left(u_{n}-\frac{\mu_{n}}{1-\alpha_{n}} F u_{n}\right)-\left(x^{*}-\frac{\mu_{n}}{1-\alpha_{n}} F x^{*}\right)\right)+\alpha_{n}\left(f\left(u_{n}\right)-x^{*}\right)\right\|^{q} \\
& \leq\left(1-\alpha_{n}\right)^{q}\left\|\left(u_{n}-\frac{\mu_{n}}{1-\alpha_{n}} F u_{n}\right)-\left(x^{*}-\frac{\mu_{n}}{1-\alpha_{n}} F x^{*}\right)\right\|^{q} \\
& \quad+q \alpha_{n}\left(1-\alpha_{n}\right)^{q-1}\left\langle f\left(u_{n}\right)-x^{*}, J_{q}\left(u_{n}-x^{*}-\frac{\mu_{n}}{1-\alpha_{n}}\left(F u_{n}-F x^{*}\right)\right)\right\rangle+\kappa_{q} \alpha_{n}^{q}\left\|f\left(u_{n}\right)-x^{*}\right\|^{q} \\
& \leq\left(1-\alpha_{n}\right)\left[\left\|u_{n}-x^{*}\right\|\left\|^{q}-\frac{\mu_{n}}{1-\alpha_{n}}\left(\beta q-\kappa_{q}\left(\frac{\mu_{n}}{1-\alpha_{n}}\right)^{q-1}\right)\right\| F u_{n}-F x^{*} \|^{q}\right] \\
& \quad+q \alpha_{n}\left(1-\alpha_{n}\right)^{q-1}\left\langle f\left(u_{n}\right)-f\left(x^{*}\right), J_{q}\left(u_{n}-x^{*}-\frac{\mu_{n}}{1-\alpha_{n}}\left(F u_{n}-F x^{*}\right)\right)\right\rangle \\
& +q \alpha_{n}\left(1-\alpha_{n}\right)^{q-1}\left\langle f\left(x^{*}\right)-x^{*}, J_{q}\left(u_{n}-x^{*}-\frac{\mu_{n}}{1-\alpha_{n}}\left(F u_{n}-F x^{*}\right)\right)\right\rangle+\kappa_{q} \alpha_{n}^{q}\left\|f\left(u_{n}\right)-x^{*}\right\|^{q} \\
& \leq\left(1-\alpha_{n}(1-q \rho)\right)\left\|u_{n}-x^{*}\right\|^{q}-\mu_{n}\left(\beta q-\kappa_{q}\left(\frac{\mu_{n}}{1-\alpha_{n}}\right)^{q-1}\right)\left\|F u_{n}-F x^{*}\right\|^{q} \\
& +q \alpha_{n}\left(1-\alpha_{n}\right)^{q-1}\left\langle f\left(x^{*}\right)-x^{*}, J_{q}\left(u_{n}-x^{*}-\frac{\mu_{n}}{1-\alpha_{n}}\left(F u_{n}-F x^{*}\right)\right)\right\rangle+\kappa_{q} \alpha_{n}^{q}\left\|f\left(u_{n}\right)-x^{*}\right\|^{q} .
\end{aligned}
$$


Using Lemma 1.3 (a), we have

$$
\begin{aligned}
& \left\|x_{n+1}-x^{*}\right\|^{q} \\
& \leq(1-\zeta)\left\|S_{n} u_{n}-x^{*}\right\|^{q}+\zeta\left\|y_{n}-x^{*}\right\|^{q}-\zeta(1-\zeta) g\left(\left\|S_{n} u_{n}-y_{n}\right\|\right) \\
& \leq(1-\zeta)\left\|u_{n}-x^{*}\right\|^{q}+\zeta\left[\left(1-\alpha_{n}(1-q \rho)\right)\left\|u_{n}-x^{*}\right\|^{q}-\mu_{n}\left(\beta q-\kappa_{q}\left(\frac{\mu_{n}}{1-\alpha_{n}}\right)^{q-1}\right)\left\|F u_{n}-F x^{*}\right\|^{q}\right. \\
& \left.+q \alpha_{n}\left(1-\alpha_{n}\right)^{q-1}\left\langle f\left(x^{*}\right)-x^{*}, J_{q}\left(u_{n}-x^{*}-\frac{\mu_{n}}{1-\alpha_{n}}\left(F u_{n}-F x^{*}\right)\right)\right\rangle+\kappa_{q} \alpha_{n}^{q}\left\|f\left(u_{n}\right)-x^{*}\right\|^{q}\right] \\
& -\zeta(1-\zeta) g\left(\left\|S_{n} u_{n}-y_{n}\right\|\right) \\
& \leq\left(1-\alpha_{n} \zeta(1-q \rho)\right)\left\|x_{n}-x^{*}\right\|^{q}-\left(1-\alpha_{n} \zeta(1-q \rho)\right)\left[r_{n} \lambda_{n}\left(\alpha q-\kappa_{q} \lambda_{n}^{q-1}\right)\left\|A x_{n}-A x^{*}\right\|^{q}\right. \\
& \left.+\lambda_{n}\left(\alpha q-\frac{\kappa_{q} \lambda_{n}^{q-1}}{r_{n}^{q-1}}\right)\left\|A v_{n}-A x^{*}\right\|^{q}\right]-\zeta \mu_{n}\left(\beta q-\kappa_{q}\left(\frac{\mu_{n}}{1-\alpha_{n}}\right)^{q-1}\right)\left\|F u_{n}-F x^{*}\right\|^{q}-\zeta(1-\zeta) \\
& \times g\left(\left\|S_{n} u_{n}-y_{n}\right\|\right)+\zeta q \alpha_{n}\left(1-\alpha_{n}\right)^{q-1}\left\langle f\left(x^{*}\right)-x^{*}, J_{q}\left(u_{n}-x^{*}-\frac{\mu_{n}}{1-\alpha_{n}}\left(F u_{n}-F x^{*}\right)\right)\right\rangle \\
& +\zeta \kappa_{q} \alpha_{n}^{q}\left\|f\left(u_{n}\right)-x^{*}\right\|^{q} .
\end{aligned}
$$

For each $n \geq 0$, we set

$$
\begin{aligned}
& \Gamma_{n}=\left\|x_{n}-x^{*}\right\|^{q}, \\
& \varepsilon_{n}=\alpha_{n} \zeta(1-q \rho), \\
& \eta_{n}=\left(1-\alpha_{n} \zeta(1-q \rho)\right)\left[r_{n} \lambda_{n}\left(\alpha q-\kappa_{q} \lambda_{n}^{q-1}\right)\left\|A x_{n}-A x^{*}\right\|^{q}+\lambda_{n}\left(\alpha q-\frac{\kappa_{q} \lambda_{n}^{q-1}}{r_{n}^{q-1}}\right)\left\|A v_{n}-A x^{*}\right\|^{q}\right] \\
& \quad+\zeta \mu_{n}\left(\beta q-\kappa_{q}\left(\frac{\mu_{n}}{1-\alpha_{n}}\right)^{q-1}\right)\left\|F u_{n}-F x^{*}\right\|^{q}+\zeta(1-\zeta) g\left(\left\|S_{n} u_{n}-y_{n}\right\|\right), \\
& \vartheta_{n}=\zeta q \alpha_{n}\left(1-\alpha_{n}\right)^{q-1}\left\langle f\left(x^{*}\right)-x^{*}, J_{q}\left(u_{n}-x^{*}-\frac{\mu_{n}}{1-\alpha_{n}}\left(F u_{n}-F x^{*}\right)\right)\right\rangle+\zeta \kappa_{q} \alpha_{n}^{q}\left\|f\left(u_{n}\right)-x^{*}\right\|^{q} .
\end{aligned}
$$

It follows that

$$
\Gamma_{n+1} \leq\left(1-\varepsilon_{n}\right) \Gamma_{n}-\eta_{n}+\vartheta_{n}, \quad \forall n \geq 0,
$$

and hence

$$
\Gamma_{n+1} \leq\left(1-\varepsilon_{n}\right) \Gamma_{n}+\vartheta_{n}, \quad \forall n \geq 0 .
$$

We next show the strong convergence of $\left\{\Gamma_{n}\right\}$ by the following two cases:

Case 1. Suppose that there exists an integer $n_{0} \geq 1$ such that $\left\{\Gamma_{n}\right\}$ is non-increasing. Then $\Gamma_{n}-\Gamma_{n+1} \rightarrow 0$. It follows that

$$
0 \leq \eta_{n} \leq \Gamma_{n}-\Gamma_{n+1}+\vartheta_{n}-\varepsilon_{n} \Gamma_{n}
$$

Since $\alpha_{n} \rightarrow 0, \varepsilon_{n} \rightarrow 0$ and $\vartheta_{n} \rightarrow 0$, we have $\eta_{n} \rightarrow 0$. This ensures that $\lim _{n \rightarrow \infty} g\left(\left\|S_{n} u_{n}-y_{n}\right\|\right)=$ 0 ,

$$
\lim _{n \rightarrow \infty}\left\|A x_{n}-A x^{*}\right\|=\lim _{n \rightarrow \infty}\left\|A v_{n}-A x^{*}\right\|=0
$$

and

$$
\lim _{n \rightarrow \infty}\left\|F u_{n}-F x^{*}\right\|=0
$$

Note that $g$ is a strictly increasing, continuous and convex function with $g(0)=0$. So, it follows that

$$
\lim _{n \rightarrow \infty}\left\|S_{n} u_{n}-y_{n}\right\|=0 .
$$

From $x_{n+1}=(1-\zeta) S_{n} u_{n}+\zeta y_{n}$, we get

$$
\lim _{n \rightarrow \infty}\left\|x_{n+1}-y_{n}\right\|=(1-\zeta) \lim _{n \rightarrow \infty}\left\|S_{n} u_{n}-y_{n}\right\|=0 .
$$


On the other hand, noticing $v_{n}=J_{\lambda_{n}}^{B}\left(x_{n}-\lambda_{n} A x_{n}\right)$ and using Lemma 1.3, we get

$$
\begin{aligned}
\left\|v_{n}-x^{*}\right\|^{q} & \leq\left\langle\left(x_{n}-\lambda_{n} A x_{n}\right)-\left(x^{*}-\lambda_{n} A x^{*}\right), J_{q}\left(v_{n}-x^{*}\right)\right\rangle \\
& \leq \frac{1}{q}\left[\left\|\left(x_{n}-\lambda_{n} A x_{n}\right)-\left(x^{*}-\lambda_{n} A x^{*}\right)\right\|^{q}+(q-1)\left\|v_{n}-x^{*}\right\|^{q}\right. \\
& \left.-h_{1}\left(\left\|x_{n}-\lambda_{n}\left(A x_{n}-A x^{*}\right)-v_{n}\right\|\right)\right]
\end{aligned}
$$

which together with Lemma 1.1 implies that

$$
\begin{aligned}
\left\|v_{n}-x^{*}\right\|^{q} & \leq\left\|\left(x_{n}-\lambda_{n} A x_{n}\right)-\left(x^{*}-\lambda_{n} A x^{*}\right)\right\|^{q}-h_{1}\left(\left\|x_{n}-\lambda_{n}\left(A x_{n}-A x^{*}\right)-v_{n}\right\|\right) \\
& \leq\left\|x_{n}-x^{*}\right\|^{q}-h_{1}\left(\left\|x_{n}-\lambda_{n}\left(A x_{n}-A x^{*}\right)-v_{n}\right\|\right) .
\end{aligned}
$$

Since $J_{\mu_{n}}^{M}$ is firmly nonexpansive, we conclude from Lemma 1.3 (c) that

$$
\begin{aligned}
\left\|y_{n}-x^{*}\right\|^{q} & \leq\left\langle\left(\alpha_{n} f\left(u_{n}\right)+\left(1-\alpha_{n}\right) u_{n}-\mu_{n} F u_{n}\right)-\left(x^{*}-\mu_{n} F x^{*}\right), J_{q}\left(y_{n}-x^{*}\right)\right\rangle \\
& \leq \frac{1}{q}\left[\left\|\left(\alpha_{n} f\left(u_{n}\right)+\left(1-\alpha_{n}\right) u_{n}-\mu_{n} F u_{n}\right)-\left(x^{*}-\mu_{n} F x^{*}\right)\right\|^{q}+(q-1)\left\|y_{n}-x^{*}\right\|^{q}\right. \\
& \left.-h\left(\left\|\alpha_{n} f\left(u_{n}\right)+\left(1-\alpha_{n}\right) u_{n}-\mu_{n}\left(F u_{n}-F x^{*}\right)-y_{n}\right\|\right)\right],
\end{aligned}
$$

which, together with the convexity of $\|\cdot\|^{q}$ and the nonexpansivity of $I-\frac{\mu_{n}}{1-\alpha_{n}} F$, implies that

$$
\begin{aligned}
\left\|y_{n}-x^{*}\right\|^{q} & \leq\left\|\left(\alpha_{n} f\left(u_{n}\right)+\left(1-\alpha_{n}\right) u_{n}-\mu_{n} F u_{n}\right)-\left(x^{*}-\mu_{n} F x^{*}\right)\right\|^{q} \\
& -h\left(\left\|\alpha_{n} f\left(u_{n}\right)+\left(1-\alpha_{n}\right) u_{n}-\mu_{n}\left(F u_{n}-F x^{*}\right)-y_{n}\right\|\right) \\
& \leq\left(1-\alpha_{n}\right)\left\|\left(u_{n}-\frac{\mu_{n}}{1-\alpha_{n}} F u_{n}\right)-\left(x^{*}-\frac{\mu_{n}}{1-\alpha_{n}} F x^{*}\right)\right\|^{q}+\alpha_{n}\left\|f\left(u_{n}\right)-x^{*}\right\|^{q} \\
& -h\left(\left\|\alpha_{n} f\left(u_{n}\right)+\left(1-\alpha_{n}\right) u_{n}-\mu_{n}\left(F u_{n}-F x^{*}\right)-y_{n}\right\|\right) \\
& \leq\left(1-\alpha_{n}\right)\left\|u_{n}-x^{*}\right\|^{q}+\alpha_{n}\left\|f\left(u_{n}\right)-x^{*}\right\|^{q} \\
& -h\left(\left\|\alpha_{n} f\left(u_{n}\right)+\left(1-\alpha_{n}\right) u_{n}-\mu_{n}\left(F u_{n}-F x^{*}\right)-y_{n}\right\|\right) .
\end{aligned}
$$

This together with (2.5) implies that

$$
\begin{aligned}
& \left\|x_{n+1}-x^{*}\right\|^{q} \leq(1-\zeta)\left\|S_{n} u_{n}-x^{*}\right\|^{q}+\zeta\left\|y_{n}-x^{*}\right\|^{q} \\
& \leq(1-\zeta)\left\|u_{n}-x^{*}\right\|^{q}+\zeta\left[\left(1-\alpha_{n}\right)\left\|u_{n}-x^{*}\right\|^{q}+\alpha_{n}\left\|f\left(u_{n}\right)-x^{*}\right\|^{q}\right. \\
& \left.-h\left(\left\|\alpha_{n} f\left(u_{n}\right)+\left(1-\alpha_{n}\right) u_{n}-\mu_{n}\left(F u_{n}-F x^{*}\right)-y_{n}\right\|\right)\right] \\
& =\left(1-\zeta \alpha_{n}\right)\left\|u_{n}-x^{*}\right\|^{q}+\zeta \alpha_{n}\left\|f\left(u_{n}\right)-x^{*}\right\|^{q} \\
& -\zeta h\left(\left\|\alpha_{n} f\left(u_{n}\right)+\left(1-\alpha_{n}\right) u_{n}-\mu_{n}\left(F u_{n}-F x^{*}\right)-y_{n}\right\|\right) \\
& \leq\left(1-\zeta \alpha_{n}\right)\left[\left(1-r_{n}\right)\left\|x_{n}-x^{*}\right\|^{q}+r_{n}\left\|v_{n}-x^{*}\right\|^{q}\right]+\zeta \alpha_{n}\left\|f\left(u_{n}\right)-x^{*}\right\|^{q} \\
& -\zeta h\left(\left\|\alpha_{n} f\left(u_{n}\right)+\left(1-\alpha_{n}\right) u_{n}-\mu_{n}\left(F u_{n}-F x^{*}\right)-y_{n}\right\|\right) \\
& \leq\left(1-\zeta \alpha_{n}\right)\left\|x_{n}-x^{*}\right\|^{q}-\left(1-\zeta \alpha_{n}\right) r_{n} h_{1}\left(\left\|x_{n}-\lambda_{n}\left(A x_{n}-A x^{*}\right)-v_{n}\right\|\right) \\
& +\zeta \alpha_{n}\left\|f\left(u_{n}\right)-x^{*}\right\|^{q}-\zeta h\left(\left\|\alpha_{n} f\left(u_{n}\right)+\left(1-\alpha_{n}\right) u_{n}-\mu_{n}\left(F u_{n}-F x^{*}\right)-y_{n}\right\|\right),
\end{aligned}
$$

which immediately yields

$$
\begin{aligned}
& \left(1-\zeta \alpha_{n}\right) r_{n} h_{1}\left(\left\|x_{n}-\lambda_{n}\left(A x_{n}-A x^{*}\right)-v_{n}\right\|\right) \\
& +\zeta h\left(\left\|\alpha_{n} f\left(u_{n}\right)+\left(1-\alpha_{n}\right) u_{n}-\mu_{n}\left(F u_{n}-F x^{*}\right)-y_{n}\right\|\right) \\
& \leq \zeta \alpha_{n}\left\|f\left(u_{n}\right)-x^{*}\right\|^{q}+\left(1-\zeta \alpha_{n}\right)\left\|x_{n}-x^{*}\right\|^{q}-\left\|x_{n+1}-x^{*}\right\|^{q} \\
& \leq \zeta \alpha_{n}\left\|f\left(u_{n}\right)-x^{*}\right\|^{q}+\Gamma_{n}-\Gamma_{n+1} .
\end{aligned}
$$

Since $h_{1}$ and $h$ are strictly increasing, continuous and convex functions with $h_{1}(0)=h(0)=0$, we conclude from $\alpha_{n} \rightarrow 0$ and $\Gamma_{n}-\Gamma_{n+1} \rightarrow 0$ that

$$
\lim _{n \rightarrow \infty}\left\|x_{n}-\lambda_{n}\left(A x_{n}-A x^{*}\right)-v_{n}\right\|=\lim _{n \rightarrow \infty}\left\|\alpha_{n} f\left(u_{n}\right)+\left(1-\alpha_{n}\right) u_{n}-\mu_{n}\left(F u_{n}-F x^{*}\right)-y_{n}\right\|=0 \text {. }
$$

Note that

$$
\left\|x_{n}-v_{n}\right\| \leq\left\|x_{n}-\lambda_{n}\left(A x_{n}-A x^{*}\right)-v_{n}\right\|+\lambda_{n}\left\|A x_{n}-A x^{*}\right\|
$$


and

$$
\begin{aligned}
\left\|u_{n}-y_{n}\right\| \leq & \left\|\alpha_{n} f\left(u_{n}\right)+\left(1-\alpha_{n}\right) u_{n}-\mu_{n}\left(F u_{n}-F x^{*}\right)-y_{n}\right\| \\
& +\alpha_{n}\left\|f\left(u_{n}\right)-u_{n}\right\|+\mu_{n}\left\|F u_{n}-F x^{*}\right\| .
\end{aligned}
$$

So it follows from (2.1), (2.2) and (2.7) that

$$
\lim _{n \rightarrow \infty}\left\|x_{n}-v_{n}\right\|=\lim _{n \rightarrow \infty}\left\|u_{n}-y_{n}\right\|=0 .
$$

In a similar way, we have

$$
\begin{aligned}
\left\|u_{n}-x^{*}\right\|^{q} \leq & \left\langle\left(x_{n}-\lambda_{n} A v_{n}+r_{n}\left(v_{n}-x_{n}\right)\right)-\left(x^{*}-\lambda_{n} A x^{*}\right), J_{q}\left(u_{n}-x^{*}\right)\right\rangle \\
\leq & \frac{1}{q}\left[\left\|\left(x_{n}-\lambda_{n} A v_{n}+r_{n}\left(v_{n}-x_{n}\right)\right)-\left(x^{*}-\lambda_{n} A x^{*}\right)\right\|^{q}+(q-1)\left\|u_{n}-x^{*}\right\|^{q}\right. \\
& \left.-h_{2}\left(\left\|x_{n}+r_{n}\left(v_{n}-x_{n}\right)-\lambda_{n}\left(A v_{n}-A x^{*}\right)-u_{n}\right\|\right)\right],
\end{aligned}
$$

which implies that

$$
\begin{aligned}
\left\|u_{n}-x^{*}\right\|^{q} & \leq\left\|\left(x_{n}-\lambda_{n} A v_{n}+r_{n}\left(v_{n}-x_{n}\right)\right)-\left(x^{*}-\lambda_{n} A x^{*}\right)\right\|^{q} \\
& -h_{2}\left(\left\|x_{n}+r_{n}\left(v_{n}-x_{n}\right)-\lambda_{n}\left(A v_{n}-A x^{*}\right)-u_{n}\right\|\right) \\
& \leq\left\|x_{n}-x^{*}\right\|^{q}-h_{2}\left(\left\|x_{n}+r_{n}\left(v_{n}-x_{n}\right)-\lambda_{n}\left(A v_{n}-A x^{*}\right)-u_{n}\right\|\right) .
\end{aligned}
$$

This together with (2.6) ensures that

$$
\begin{aligned}
\left\|x_{n+1}-x^{*}\right\|^{q} & \leq(1-\zeta)\left\|S_{n} u_{n}-x^{*}\right\|^{q}+\zeta\left\|y_{n}-x^{*}\right\|^{q} \\
& \leq(1-\zeta)\left\|u_{n}-x^{*}\right\|^{q}+\zeta\left[\left(1-\alpha_{n}\right)\left\|u_{n}-x^{*}\right\|^{q}+\alpha_{n}\left\|f\left(u_{n}\right)-x^{*}\right\|^{q}\right] \\
& \leq\left(1-\zeta \alpha_{n}\right)\left[\left\|x_{n}-x^{*}\right\|^{q}-h_{2}\left(\left\|x_{n}+r_{n}\left(v_{n}-x_{n}\right)-\lambda_{n}\left(A v_{n}-A x^{*}\right)-u_{n}\right\|\right)\right] \\
& +\zeta \alpha_{n}\left\|f\left(u_{n}\right)-x^{*}\right\|^{q} \\
& \leq\left\|x_{n}-x^{*}\right\|^{q}-\left(1-\zeta \alpha_{n}\right) h_{2}\left(\left\|x_{n}+r_{n}\left(v_{n}-x_{n}\right)-\lambda_{n}\left(A v_{n}-A x^{*}\right)-u_{n}\right\|\right) \\
& +\zeta \alpha_{n}\left\|f\left(u_{n}\right)-x^{*}\right\|^{q} .
\end{aligned}
$$

So, it follows that

$$
\left(1-\zeta \alpha_{n}\right) h_{2}\left(\left\|x_{n}+r_{n}\left(v_{n}-x_{n}\right)-\lambda_{n}\left(A v_{n}-A x^{*}\right)-u_{n}\right\|\right) \leq \Gamma_{n}-\Gamma_{n+1}+\zeta \alpha_{n}\left\|f\left(u_{n}\right)-x^{*}\right\|^{q} .
$$

Since $h_{2}$ is a strictly increasing, continuous and convex function with $h_{2}(0)=0$, we find from $\alpha_{n} \rightarrow 0$ and $\Gamma_{n}-\Gamma_{n+1} \rightarrow 0$ that

$$
\lim _{n \rightarrow \infty}\left\|x_{n}+r_{n}\left(v_{n}-x_{n}\right)-\lambda_{n}\left(A v_{n}-A x^{*}\right)-u_{n}\right\|=0 .
$$

Observe that

$$
\begin{aligned}
\left\|x_{n}-u_{n}\right\| & =\left\|x_{n}+r_{n}\left(v_{n}-x_{n}\right)-\lambda_{n}\left(A v_{n}-A x^{*}\right)-u_{n}-r_{n}\left(v_{n}-x_{n}\right)+\lambda_{n}\left(A v_{n}-A x^{*}\right)\right\| \\
& \leq\left\|x_{n}+r_{n}\left(v_{n}-x_{n}\right)-\lambda_{n}\left(A v_{n}-A x^{*}\right)-u_{n}\right\|+r_{n}\left\|v_{n}-x_{n}\right\|+\lambda_{n}\left\|A v_{n}-A x^{*}\right\| .
\end{aligned}
$$

So it follows from (2.1), (2.8) and (2.9) that

$$
\lim _{n \rightarrow \infty}\left\|x_{n}-u_{n}\right\|=0
$$

This together with (2.4) and (2.8) leads to

$$
\left\|x_{n+1}-x_{n}\right\| \leq\left\|x_{n+1}-y_{n}\right\|+\left\|y_{n}-u_{n}\right\|+\left\|u_{n}-x_{n}\right\| \rightarrow 0 \quad(n \rightarrow \infty) .
$$

On the other hand, taking into account

$$
\begin{aligned}
\left\|S_{n} x_{n}-x_{n}\right\| & \leq\left\|S_{n} x_{n}-S_{n} u_{n}\right\|+\left\|S_{n} u_{n}-y_{n}\right\|+\left\|y_{n}-u_{n}\right\|+\left\|u_{n}-x_{n}\right\| \\
& \leq 2\left\|x_{n}-u_{n}\right\|+\left\|S_{n} u_{n}-y_{n}\right\|+\left\|y_{n}-u_{n}\right\|,
\end{aligned}
$$


we deduce from (2.3), (2.8) and (2.10) that $\lim _{n \rightarrow \infty}\left\|S_{n} x_{n}-x_{n}\right\|=0$. Using Lemma 1.4 and the assumption on $\left\{S_{n}\right\}_{n=0}^{\infty}$, we get $\lim _{n \rightarrow \infty}\left\|S_{n} x_{n}-S x_{n}\right\|=0$. Therefore, we conclude that

$$
\left\|x_{n}-S x_{n}\right\| \leq\left\|x_{n}-S_{n} x_{n}\right\|+\left\|S_{n} x_{n}-S x_{n}\right\| \rightarrow 0 \quad(n \rightarrow \infty) .
$$

For each $n \geq 0$, we put $T_{\lambda_{n}}:=J_{\lambda_{n}}^{B}\left(I-\lambda_{n} A\right)$. It follows from (2.8) that

$$
\lim _{n \rightarrow \infty}\left\|x_{n}-T_{\lambda_{n}} x_{n}\right\|=\lim _{n \rightarrow \infty}\left\|x_{n}-v_{n}\right\|=0 .
$$

Noticing $0<\lambda \leq \lambda_{n}$ for all $n \geq 0$ and using Lemma 1.5 (ii), we obtain

$$
\left\|T_{\lambda} x_{n}-x_{n}\right\| \leq 2\left\|T_{\lambda_{n}} x_{n}-x_{n}\right\| \rightarrow 0 \quad(n \rightarrow \infty) .
$$

In addition, for each $n \geq 0$, we put $T_{\mu_{n}}:=J_{\mu_{n}}^{M}\left(I-\mu_{n} F\right)$. From (2.8) and $\alpha_{n} \rightarrow 0$, we get

$$
\begin{aligned}
\left\|u_{n}-T_{\mu_{n}} u_{n}\right\| & \leq\left\|u_{n}-J_{\mu_{n}}^{M}\left(\alpha_{n} f\left(u_{n}\right)+\left(1-\alpha_{n}\right) u_{n}-\mu_{n} F u_{n}\right)\right\| \\
& +\left\|J_{\mu_{n}}^{M}\left(\alpha_{n} f\left(u_{n}\right)+\left(1-\alpha_{n}\right) u_{n}-\mu_{n} F u_{n}\right)-J_{\mu_{n}}^{M}\left(u_{n}-\mu_{n} F u_{n}\right)\right\| \\
& \leq\left\|u_{n}-y_{n}\right\|+\alpha_{n}\left\|f\left(u_{n}\right)-u_{n}\right\| \rightarrow 0 \quad(n \rightarrow \infty) .
\end{aligned}
$$

Since $\lim _{n \rightarrow \infty} a\left(1-\alpha_{n}\right)=a>0$, without loss of generality, we may assume that $\exists \mu>0$ such that $\mu \leq a\left(1-\alpha_{n}\right) \leq \mu_{n} \forall n \geq 0$. Using Lemma 1.5 (ii), we obtain from (2.10) that

$$
\begin{aligned}
\left\|T_{\mu} x_{n}-x_{n}\right\| & \leq\left\|T_{\mu} x_{n}-T_{\mu} u_{n}\right\|+\left\|T_{\mu} u_{n}-u_{n}\right\|+\left\|u_{n}-x_{n}\right\| \\
& \leq 2\left\|x_{n}-u_{n}\right\|+\left\|T_{\mu} u_{n}-u_{n}\right\| \\
& \leq 2\left\|x_{n}-u_{n}\right\|+2\left\|T_{\mu_{n}} u_{n}-u_{n}\right\| \rightarrow 0 \quad(n \rightarrow \infty) .
\end{aligned}
$$

We now define a mapping $\Psi: C \rightarrow C$ by $\Psi x:=v_{1} S x+v_{2} T_{\lambda} x+\left(1-v_{1}-v_{2}\right) T_{\mu} x, \forall x \in C$ with $v_{1}+v_{2}<1$ for constants $v_{1}, v_{2} \in(0,1)$. From Lemma 1.5 (i) and Lemma 1.6, we know that $\Psi$ is nonexpansive and

$$
\operatorname{Fix}(\Psi)=\operatorname{Fix}(S) \cap \operatorname{Fix}\left(T_{\lambda}\right) \cap \operatorname{Fix}\left(T_{\mu}\right)=\bigcap_{n=0}^{\infty} \operatorname{Fix}\left(S_{n}\right) \cap(A+B)^{-1} 0 \cap(F+M)^{-1} 0(=: \Omega) .
$$

Taking into account

$$
\left\|\Psi x_{n}-x_{n}\right\| \leq v_{1}\left\|S x_{n}-x_{n}\right\|+v_{2}\left\|T_{\lambda} x_{n}-x_{n}\right\|+\left(1-v_{1}-v_{2}\right)\left\|T_{\mu} x_{n}-x_{n}\right\|,
$$

we deduce from (2.11)-(2.13) that

$$
\lim _{n \rightarrow \infty}\left\|\Psi x_{n}-x_{n}\right\|=0 .
$$

Let $z_{t}=t f\left(z_{t}\right)+(1-t) \Psi z_{t} \forall t \in(0,1)$. Then it follows from Lemma 1.7 that $\left\{z_{t}\right\}$ converges strongly to a point $x^{*} \in \operatorname{Fix}(\Psi)=\Omega$, which solves

$$
\left\langle(I-f) x^{*}, J\left(x^{*}-p\right)\right\rangle \leq 0, \quad \forall p \in \Omega .
$$

It follows that

$$
\begin{aligned}
\left\|z_{t}-x_{n}\right\|^{q} & \leq(1-t)^{q}\left\|\Psi z_{t}-x_{n}\right\|^{q}+q t\left\langle f\left(z_{t}\right)-x_{n}, J_{q}\left(z_{t}-x_{n}\right)\right\rangle \\
& \leq(1-t)^{q}\left(\left\|\Psi z_{t}-\Psi x_{n}\right\|+\left\|\Psi x_{n}-x_{n}\right\|\right)^{q}+q t\left\langle f\left(z_{t}\right)-z_{t}, J_{q}\left(z_{t}-x_{n}\right)\right\rangle+q t\left\|z_{t}-x_{n}\right\|^{q} \\
& \leq(1-t)^{q}\left(\left\|z_{t}-x_{n}\right\|+\left\|\Psi x_{n}-x_{n}\right\|\right)^{q}+q t\left\langle f\left(z_{t}\right)-z_{t}, J_{q}\left(z_{t}-x_{n}\right)\right\rangle+q t\left\|z_{t}-x_{n}\right\|^{q},
\end{aligned}
$$

which immediately attains

$$
\left\langle f\left(z_{t}\right)-z_{t}, J_{q}\left(x_{n}-z_{t}\right)\right\rangle \leq \frac{(1-t)^{q}}{q t}\left(\left\|z_{t}-x_{n}\right\|+\left\|\Psi x_{n}-x_{n}\right\|\right)^{q}+\frac{q t-1}{q t}\left\|z_{t}-x_{n}\right\|^{q} .
$$


From (2.14), we have

$$
\limsup _{n \rightarrow \infty}\left\langle f\left(z_{t}\right)-z_{t}, J_{q}\left(x_{n}-z_{t}\right)\right\rangle \leq \frac{(1-t)^{q}}{q t} K+\frac{q t-1}{q t} K=\left(\frac{(1-t)^{q}+q t-1}{q t}\right) K,
$$

where $K$ is a constant such that $\left\|z_{t}-x_{n}\right\|^{q} \leq K$ for all $n \geq 0$ and $t \in(0,1)$. It is clear that $\left((1-t)^{q}+q t-1\right) / q t \rightarrow 0$ as $t \rightarrow 0$. Since $J_{q}$ is norm-to-norm uniformly continuous on bounded subsets of $E$ and $z_{t} \rightarrow x^{*}$, we get $\left\|J_{q}\left(x_{n}-z_{t}\right)-J_{q}\left(x_{n}-x^{*}\right)\right\| \rightarrow 0$ as $t \rightarrow 0$. So, we obtain

$$
\begin{aligned}
& \left|\left\langle f\left(z_{t}\right)-z_{t}, J_{q}\left(x_{n}-z_{t}\right)\right\rangle-\left\langle f\left(x^{*}\right)-x^{*}, J_{q}\left(x_{n}-x^{*}\right)\right\rangle\right| \\
& \leq\left|\left\langle f\left(x^{*}\right)-x^{*}, J_{q}\left(x_{n}-z_{t}\right)-J_{q}\left(x_{n}-x^{*}\right)\right\rangle\right|+\left|\left\langle f\left(z_{t}\right)-f\left(x^{*}\right), J_{q}\left(x_{n}-z_{t}\right)\right\rangle\right| \\
& +\left|\left\langle x^{*}-z_{t}, J_{q}\left(x_{n}-z_{t}\right)\right\rangle\right| \\
& \leq\left\|f\left(x^{*}\right)-x^{*}\right\|\left\|J_{q}\left(x_{n}-z_{t}\right)-J_{q}\left(x_{n}-x^{*}\right)\right\|+(1+\rho)\left\|z_{t}-x^{*}\right\|\left\|x_{n}-z_{t}\right\|^{q-1} .
\end{aligned}
$$

Thus, for each $n \geq 0$, we have

$$
\lim _{t \rightarrow 0}\left\langle f\left(z_{t}\right)-z_{t}, J_{q}\left(x_{n}-z_{t}\right)\right\rangle=\left\langle f\left(x^{*}\right)-x^{*}, J_{q}\left(x_{n}-x^{*}\right)\right\rangle .
$$

From (2.15), as $t \rightarrow 0$, it follows that

$$
\limsup _{n \rightarrow \infty}\left\langle f\left(x^{*}\right)-x^{*}, J_{q}\left(x_{n}-x^{*}\right)\right\rangle \leq 0 .
$$

By (C3), (2.1) and (2.10), we get

$$
\begin{aligned}
\left\|u_{n}-x^{*}-\frac{\mu_{n}}{1-\alpha_{n}}\left(F u_{n}-F x^{*}\right)-\left(x_{n}-x^{*}\right)\right\| & \leq\left\|u_{n}-x_{n}\right\|+\frac{\mu_{n}}{1-\alpha_{n}}\left\|F u_{n}-F x^{*}\right\| \\
& \leq\left\|u_{n}-x_{n}\right\|+b\left\|F u_{n}-F x^{*}\right\| \rightarrow 0 \quad(n \rightarrow \infty) .
\end{aligned}
$$

Using (2.16) and (2.17), we have

$$
\limsup _{n \rightarrow \infty}\left\langle f\left(x^{*}\right)-x^{*}, J_{q}\left(u_{n}-x^{*}-\frac{\mu_{n}}{1-\alpha_{n}}\left(F u_{n}-F x^{*}\right)\right)\right\rangle \leq 0 .
$$

It follows from (2.8) that

$$
\begin{aligned}
& \left\|x_{n+1}-x^{*}\right\|^{q} \leq\left(1-\alpha_{n} \zeta(1-q \rho)\right)\left\|x_{n}-x^{*}\right\|^{q} \\
& +\zeta q \alpha_{n}\left(1-\alpha_{n}\right)^{q-1}\left\langle f\left(x^{*}\right)-x^{*}, J_{q}\left(u_{n}-x^{*}-\frac{\mu_{n}}{1-\alpha_{n}}\left(F u_{n}-F x^{*}\right)\right)\right\rangle+\zeta \kappa_{q} \alpha_{n}^{q}\left\|f\left(u_{n}\right)-x^{*}\right\|^{q} \\
& =\left(1-\alpha_{n} \zeta(1-q \rho)\right)\left\|x_{n}-x^{*}\right\|^{q} \\
& +\alpha_{n} \zeta(1-q \rho)\left[\frac{q\left(1-\alpha_{n}\right)^{q-1}\left\langle f\left(x^{*}\right)-x^{*}, J_{q}\left(u_{n}-x^{*}-\frac{\lambda_{n}}{1-\alpha_{n}}\left(A u_{n}-A x^{*}\right)\right)\right\rangle}{1-q \rho}+\frac{\kappa_{q} \alpha_{n}^{q-1}\left\|f\left(u_{n}\right)-x^{*}\right\|^{q}}{1-q \rho}\right] .
\end{aligned}
$$

Note that $\left\{\alpha_{n} \zeta(1-q \rho)\right\} \subset[0,1], \sum_{n=0}^{\infty} \alpha_{n} \zeta(1-q \rho)=\infty$ and

$$
\limsup _{n \rightarrow \infty}\left[\frac{q\left(1-\alpha_{n}\right)^{q-1}\left\langle f\left(x^{*}\right)-x^{*}, J_{q}\left(u_{n}-x^{*}-\frac{\lambda_{n}}{1-\alpha_{n}}\left(A u_{n}-A x^{*}\right)\right)\right\rangle}{1-q \rho}+\frac{\kappa_{q} \alpha_{n}^{q-1}\left\|f\left(u_{n}\right)-x^{*}\right\|^{q}}{1-q \rho}\right] \leq 0 .
$$

Applying Lemma 1.8 to (2.18), we deduce that $\Gamma_{n} \rightarrow 0$ as $n \rightarrow \infty$. Thus, $x_{n} \rightarrow x^{*}$ as $n \rightarrow \infty$.

Case 2. Suppose that there exits $\left\{\Gamma_{m_{l}}\right\} \subset\left\{\Gamma_{m}\right\}$ such that $\Gamma_{m_{l}}<\Gamma_{m_{l}+1}, \forall l \in \mathbb{N}$, where $\mathbb{N}$ is the set of all positive integers. Define a mapping $\tau: \mathbb{N} \rightarrow \mathbb{N}$ by

$$
\tau(m):=\max \left\{l \leq m: \Gamma_{l}<\Gamma_{l+1}\right\} .
$$

Using Lemma 1.9, we have

$$
\Gamma_{\tau(m)} \leq \Gamma_{\tau(m)+1} \quad \text { and } \quad \Gamma_{m} \leq \Gamma_{\tau(m)+1} .
$$


Putting $\Gamma_{m}=\left\|x_{m}-x^{*}\right\|^{q}, \forall m \in \mathbb{N}$ and using the same inference as in Case 1, we can obtain

$$
\lim _{m \rightarrow \infty}\left\|x_{\tau(m)+1}-x_{\tau(m)}\right\|=0
$$

and

$$
\limsup _{m \rightarrow \infty}\left\langle f\left(x^{*}\right)-x^{*}, J_{q}\left(u_{\tau(m)}-x^{*}-\frac{\mu_{\tau(m)}}{1-\alpha_{\tau(m)}}\left(F u_{\tau(m)}-F x^{*}\right)\right)\right\rangle \leq 0 .
$$

In view of $\Gamma_{\tau(m)} \leq \Gamma_{\tau(m)+1}$ and $\alpha_{\tau(m)}>0$, we conclude that

$$
\begin{aligned}
\left\|x_{\tau(m)}-x^{*}\right\|^{q} \leq & \frac{q\left(1-\alpha_{\tau(m)}\right)^{q-1}}{1-q \rho}\left\langle f\left(x^{*}\right)-x^{*}, J_{q}\left(u_{\tau(m)}-x^{*}-\frac{\mu_{\tau(m)}}{1-\alpha_{\tau(m)}}\left(F u_{\tau(m)}-F x^{*}\right)\right)\right\rangle \\
& +\frac{\kappa_{q} \alpha_{\tau(m)}^{q-1}}{1-q \rho}\left\|f\left(u_{\tau(m)}\right)-x^{*}\right\|^{q},
\end{aligned}
$$

and hence $\limsup _{m \rightarrow \infty}\left\|x_{\tau(m)}-x^{*}\right\|^{q} \leq 0$. Thus, we have $\lim _{m \rightarrow \infty}\left\|x_{\tau(m)}-x^{*}\right\|^{q}=0$. Using Lemma 1.2 and (2.19), we obtain

$$
\begin{aligned}
& \left\|x_{\tau(m)+1}-x^{*}\right\|^{q}-\left\|x_{\tau(m)}-x^{*}\right\|^{q} \\
& \leq q\left\langle x_{\tau(m)+1}-x_{\tau(m)}, J_{q}\left(x_{\tau(m)}-x^{*}\right)\right\rangle+\kappa_{q}\left\|x_{\tau(m)+1}-x_{\tau(m)}\right\|^{q} \\
& \leq q\left\|x_{\tau(m)+1}-x_{\tau(m)}\right\|\left\|x_{\tau(m)}-x^{*}\right\|^{q-1}+\kappa_{q}\left\|x_{\tau(m)+1}-x_{\tau(m)}\right\|^{q} \rightarrow 0 \quad(m \rightarrow \infty) .
\end{aligned}
$$

Taking into account $\Gamma_{m} \leq \Gamma_{\tau(m)+1}$, we have

$$
\left\|x_{m}-x^{*}\right\|^{q} \leq\left\|x_{\tau(m)}-x^{*}\right\|^{q}+q\left\|x_{\tau(m)+1}-x_{\tau(m)}\right\|\left\|x_{\tau(m)}-x^{*}\right\|^{q-1}+\kappa_{q}\left\|x_{\tau(m)+1}-x_{\tau(m)}\right\|^{q} .
$$

It is easy to see from (2.19) that $x_{m} \rightarrow x^{*}$ as $m \rightarrow \infty$. This completes the proof.

In the framework of Hilbert spaces, we have the following result immediately.

Corollary 2.1. Let $C$ be a closed convex nonempty subset of a real Hilbert space $H$. Let $f$ : $C \rightarrow C$ be a $\rho$-contraction with constant $\rho \in\left[0, \frac{1}{q}\right)$, and let $\left\{S_{n}\right\}_{n=0}^{\infty}$ be a countable family of nonexpansive self-mappings on $C$. Suppose that $B, M: C \rightarrow 2^{H}$ are both maximal monotone operators, and $A, F: C \rightarrow E$ are $\alpha$-inverse-strongly monotone mapping and $\beta$-inverse-strongly monotone mapping, respectively. Assume that $\Omega:=\bigcap_{n=0}^{\infty} \operatorname{Fix}\left(S_{n}\right) \cap(A+B)^{-1} 0 \cap(F+M)^{-1} 0 \neq$ $\emptyset$. For any given $x_{0} \in C$ and $\zeta \in(0,1)$, let $\left\{x_{n}\right\}_{n=0}^{\infty}$ be the sequence generated by

$$
\left\{\begin{array}{l}
v_{n}=J_{\lambda_{n}}^{B}\left(x_{n}-\lambda_{n} A x_{n}\right), \\
u_{n}=J_{\lambda_{n}}^{B}\left(x_{n}-\lambda_{n} A v_{n}+r_{n}\left(v_{n}-x_{n}\right)\right), \\
x_{n+1}=(1-\zeta) S_{n} u_{n}+\zeta J_{\mu_{n}}^{M}\left(\alpha_{n} f\left(u_{n}\right)+\left(1-\alpha_{n}\right) u_{n}-\mu_{n} F u_{n}\right), \quad \forall n \geq 0,
\end{array}\right.
$$

where the sequences $\left\{r_{n}\right\},\left\{\alpha_{n}\right\} \subset(0,1)$ and $\left\{\lambda_{n}\right\},\left\{\mu_{n}\right\} \subset(0, \infty)$ are such that the following conditions hold: (C1) $\lim _{n \rightarrow \infty} \alpha_{n}=0$ and $\sum_{n=0}^{\infty} \alpha_{n}=\infty$; (C2) $0<r \leq r_{n}<1$ and $0<\lambda \leq \lambda_{n}<$ $\frac{\lambda_{n}}{r_{n}} \leq \bar{\lambda}<2 \alpha$; (C3) $0<a \leq \frac{\mu_{n}}{1-\alpha_{n}} \leq b<2 \beta$. Assume that $\sum_{n=0}^{\infty} \sup _{x \in D}\left\|S_{n+1} x-S_{n} x\right\|<\infty$ for any bounded subset $D$ of $C$. Let $S: C \rightarrow C$ be a mapping defined by $S x=\lim _{n \rightarrow \infty} S_{n} x \forall x \in C$, and suppose that $\operatorname{Fix}(S)=\bigcap_{n=0}^{\infty} \operatorname{Fix}\left(S_{n}\right)$. Then $x_{n} \rightarrow x^{*} \in \Omega$, which is the unique solution to $\left\langle(I-f) x^{*}, p-x^{*}\right\rangle \geq 0, \forall p \in \Omega$, i.e., the fixed point equation $x^{*}=P_{\Omega} f\left(x^{*}\right)$.

\section{Acknowledgments}

This paper was partially supported by the Innovation Program of Shanghai Municipal Education Commission (15ZZ068), Ph.D. Program Foundation of Ministry of Education of China (20123127110002) and Program for Outstanding Academic Leaders in Shanghai City (15XD150 $3100)$. 


\section{REFERENCES}

[1] Y. Shehu, J.C. Yao, Rate of convergence for inertial iterative method for countable family of certain quasinonexpansive mappings, J. Nonlinear Convex Anal. 21 (2020), 533-541.

[2] X. Qin, N.T. An, Smoothing algorithms for computing the projection onto a Minkowski sum of convex sets, Comput. Optim. Appl. 74 (2019), 821-850.

[3] G. Kassay, S. Reich, S. Sabach, Iterative methods for solving systems of variational inequalities in reflexive Banach spaces, SIAM J. Optim. 21 (2011), 131901344.

[4] L.C. Ceng, Z.R. Kong, C.F. Wen, On general systems of variational inequalities, Comput. Math. Appl. 66 (2013), 1514-1532.

[5] L.C. Ceng, C.T. Pang, C.F. Wen, Implicit and explicit iterative methods for mixed equilibria with constraints of system of generalized equilibria and hierarchical fixed point problem, J. Inequal. Appl. 2015 (2015), 280.

[6] S.Y. Cho, A convergence theorem for generalized mixed equilibrium problems and multivalued asymptotically nonexpansive mappings, J. Nonlinear Convex Anal. 21 (2020), 1017-1026.

[7] K. Goebel, S. Reich, Uniform Convexity, Hyperbolic Geometry, and Nonexpansive Mappings, Dekker, New York and Basel, 1984.

[8] H. Zhou, X. Qin, Fixed Points of Nonlinear Operators. Iterative Methods, De Gruyter, Berlin, 2020.

[9] H.K. Xu, Inequalities in Banach spaces with applications, Nonlinear Anal. 16 (1991), 1127-1138

[10] V. Barbu, Nonlinear Semigroups and Differential Equations in Banach Spaces, Noordhoff, Amsterdam, 1976.

[11] S.S. Chang, C.F. Wen, J.C. Yao, Generalized viscosity implicit rules for solving quasi- inclusion problems of accretive operators in Banach spaces, Optimization 66 (2017), 1105-1117.

[12] P. Sunthrayuth, P. Cholamjiak, A modified extragradient method for variational inclusion and fixed point problems in Banach spaces, Appl. Anal. (2019), doi: 10.1080/00036811.2019.1673374.

[13] X. Qin, S.Y. Cho, J.C. Yao, Weak and strong convergence of splitting algorithms in Banach spaces, Optimization, 69 (2020), 243-267.

[14] A.A.N. Abdou, B.A.S. Alamri, Y.J. Cho, Y. Yao, L.J. Zhu, Parallel algorithms for variational inclusions and fixed points with applications, Fixed Point Theory Appl. 2014 (2014), 174.

[15] S.S. Chang, C.F. Wen, J.C. Yao, Common zero point for a finite family of inclusion problems of accretive mappings in Banach spaces, Optimization 67 (2018), 1183-1196.

[16] X. Qin, S.Y. Cho, L. Wang, Iterative algorithms with errors for zero points of m-accretive operators, Fixed Point Theory Appl. 2013 (2013), Article ID 148.

[17] X. Qin, S.Y. Cho, L. Wang, Strong convergence of an iterative algorithm involving nonlinear mappings of nonexpansive and accretive type, Optimization, 67 (2018), 1377-1388.

[18] K. Aoyama, Y. Kimura, W. Takahashi, M. Toyoda, Approximation of common fixed points of a countable family of nonexpansive mappings in a Banach space, Nonlinear Anal. 67 (2007), 2350-2360.

[19] G. Lopez, V. Martin-Marquez, F. Wang, H.K. Xu, Forward-backward splitting methods for accretive operators in Banach spaces, Abstr. Appl. Anal. 2012 (2012), Article ID 109236.

[20] R.E. Bruck, Properties of fixed-point sets of nonexpansive mappings in Banach spaces, Trans. Amer. Math. Soc. 179 (1973), 251-262.

[21] H.K. Xu, Viscosity approximation methods for nonexpansive mappings, J. Math. Anal. Appl. 298 (2004), 279-291.

[22] H.K. Xu, Iterative algorithms for nonlinear operators, J. London Math. Soc. 66 (2002), 240-256.

[23] P.E. Maingé, Strong convergence of projected subgradient methods for nonsmooth and nonstrictly convex minimization, Set-Valued Anal. 16 (2008), 899-912. 